\title{
Time-resolved photoluminescence study of silica nanoparticles as compared to bulk type-III fused silica
}

\author{
Yuri D. Glinka* \\ Institute of Atomic and Molecular Sciences, Academia Sinica, P.O. Box 23-166, Taipei 106, Taiwan, R.O.C. \\ and Institute of Surface Chemistry of the National Academy of Sciences of Ukraine, Prosp. Nauki 31, Kiev 252650, Ukraine \\ Sheng-Hsien Lin and Yit-Tsong Chen \\ Institute of Atomic and Molecular Sciences, Academia Sinica, P.O. Box 23-166, Taipei 106, Taiwan, R.O.C. \\ and Department of Chemistry, National Taiwan University, Taipei 106, Taiwan, R.O.C. \\ (Received 10 August 2001; revised manuscript received 27 December 2001; published 3 July 2002)
}

Photoluminescence (PL) from composites of 7- and 15-nm silica (amorphous $\mathrm{SiO}_{2}$ ) nanoparticles and bulk type-III fused silica induced by two-photon band-to-band excitation with 193-nm (6.4-eV) ArF laser light has been measured in time-resolved detection mode. The PL spectra taken for 15-nm particles allow one to identify three PL bands peaked in the red $(\sim 1.9 \mathrm{eV})$, green $(\sim 2.35 \mathrm{eV})$, and blue $(\sim 2.85 \mathrm{eV})$ spectral ranges. The green and blue bands are normally overlapped in conventional scan measurements, giving no way for determining their exact peak positions. Similar red and green PL bands were observed for 7-nm particles, whereas the blue band extends toward the higher-energy range and is peaked at $\sim 3.25 \mathrm{eV}$. The aforementioned red, green, and blue PL bands are assigned to nonbridging oxygen hole centers, hydrogen-related species, and self-trapped excitons (STE's), respectively. The red and green PL bands for bulk type-III fused silica are peaked at practically the same spectral positions as those for nanoscale silicas, indicating the similarity of light-emitter types. However, the blue band for bulk silica used is peaked at $\sim 2.75 \mathrm{eV}$, that is, at typical position for STE's in crystalline $\mathrm{SiO}_{2}$. The blueshift of STE (PL) (STEPL) band with decreasing nanoparticle size is consistent with the previously proposed model of phonon-assisted radiative relaxation of STE's [Yu. D. Glinka et al., Phys. Rev. B 64, 085421 (2001)]. As a result of time-resolved measurements, the extremely broad PL band peaked at $\sim 2.35 \mathrm{eV}$, which is typically observed for bulk silicas and initially assigned to STE's, is found to consist of two bands peaked at $\sim 2.75$ and $\sim 2.35 \mathrm{eV}$. We suggest that these bands are due to the radiative deexcitation of STE's and hydrogen-related centers, respectively. We thus conclude that the STEPL band is peaked at $\sim 2.75 \mathrm{eV}$ for both bulk amorphous and crystalline $\mathrm{SiO}_{2}$.

DOI: 10.1103/PhysRevB.66.035404

PACS number(s): 81.07.-b, 78.55.Hx, 61.46.+w, 42.62.Fi

\section{INTRODUCTION}

A recent study of two-photon (TP) excited photoluminescence (PL) from silica (amorphous $\mathrm{SiO}_{2}$ ) nanoparticles induced by band-to-band excitation with 6.4-eV ArF laser light revealed the complicated dynamics of free excitons (FE's) in the confined space of nanometer-sized wide-band-gap materials. ${ }^{1-3}$ Because of the small radius of FE's, they travel through the lattice and transfer energy to impurities and structure defects. Additionally, FE's suffer collisions with the nanoparticle boundary since their mean free path length in bulk silicas is much longer than the size of nanoparticles used. ${ }^{2,3}$ As a result, FE's can be scattered by the boundary either elastically (without energy transfer) or nonelastically (with energy transfer). In a particular case of elastic scattering, the laser heating of FE's becomes dominant, resulting in a highly heated electron-hole plasma, which after cooling is condensed into self-trapped exciton (STE) and self-trapped biexciton (STBE) states. ${ }^{2,3}$ The further radiative relaxation of STE's gives rise to the STE (PL) (STEPL) band, which has been found to peak in the blue spectral range. ${ }^{2,3}$ The radiationless relaxation of STBE's leads to Frenkel defect formation. ${ }^{2}$ The STEPL band is extended and shifted toward the higher-energy range when the size of nanoparticles decreases. The latter behavior was suggested to result from the activation of phonon-assisted transitions within STE radia- tive relaxation. ${ }^{3}$ In conventional scan measurements the STEPL band is normally overlapped with the green PL band assigned to hydrogen-related centers, so its exact peak position is poorly detectable. ${ }^{1,2}$

A similar blue PL band was previously reported for bulk crystalline and amorphous $\mathrm{SiO}_{2} \cdot{ }^{4-9}$ It is associated with all STE's, twofold-coordinated $\mathrm{Si}$, or oxygen vacancies. ${ }^{4-9}$ To summarize previous findings, we note that several authors argued that for crystalline $\mathrm{SiO}_{2}$ the STEPL band is peaked between 2.6 and $2.8 \mathrm{eV}^{4-8}$ whereas in amorphous materials it extends over the entire visible spectral range with a maximum between 2.2 and $2.4 \mathrm{eV}^{8,10-13}$ Relying on such experimental data, the conclusion has been made that the spectroscopic difference in the peak positions of the STEPL band reflects the specific features of self-trapped exciton formation in crystalline and amorphous $\mathrm{SiO}_{2}{ }^{8,10-13}$ By contrast, the peak position of the STEPL band measured for silica nanoparticles is reported to be close to that observed for bulk crystalline $\mathrm{SiO}_{2} .{ }^{1-3}$ The controversy regarding the assignment of STEPL induced with band-to-band excitation in amorphous $\mathrm{SiO}_{2}$ materials is likely to originate from the extremely large width of the STEPL band, which is additionally overlapped with others originated from defect centers. Assuming that light emitters contributing to PL from $\mathrm{SiO}_{2}$ materials are characterized by different lifetimes, one can expect that the exact peak position of the STEPL band can 
exclusively be extracted using time-resolved techniques.

In this paper we present a thorough study of time-resolved band-to-band-excited PL from silica nanoparticles in comparison with that from bulk silica. The PL was induced by TP excitation with 193-nm (6.4-eV) ArF laser light. The timeresolved PL spectra for nanoscale silicas reveal three PL bands peaked in the red $(\sim 1.9)$, green $(\sim 2.35)$, and blue $(2.85-3.25 \mathrm{eV})$ spectral ranges, which were assigned to nonbridging oxygen hole centers (NBOHC's), hydrogen-related species, and STE's, respectively. The bulk type-III fused silica, which is known to be a hydrogen-contained material, ${ }^{14}$ was used in our measurements for comparison. Since the amorphous $\mathrm{SiO}_{2}$ nanoparticles are characterized by a large concentration of hydrogen-related species as well, ${ }^{1,2}$ the PL bands originated from hydrogen-related centers and NBOHC's are found to be similar to those for bulk silica. Alternatively, the size of nanoparticles exclusively affects the position of the STEPL band. The blueshift of the STEPL band with decreasing nanoparticle size is consistent with the previously proposed model of phonon-assisted radiative relaxation of STE's. ${ }^{3}$ The STEPL band for bulk silica is found to peak at $\sim 2.75 \mathrm{eV}$, and so it occupies spectral position close to that typically observed for crystalline $\mathrm{SiO}_{2}$. This fact points to the independence of STE energetic parameters on the flexibility of both $\mathrm{SiO}_{4}$ tetrahedron torsion and $\mathrm{Si}$ $\mathrm{O}-\mathrm{Si}$ angles in three-dimensional $\mathrm{SiO}_{2}$ network.

\section{EXPERIMENT}

Two kinds of silica nanoparticles (Aerosil, Degussa) with nominal particle sizes of 7 and $15 \mathrm{~nm}$ (diameter) were studied. The powders were pressed into pellets and then heat treated at $T_{\mathrm{ht}}=1173 \mathrm{~K}$ for $2 \mathrm{~h}$ in air. The PL measurements were performed in a vacuum chamber at 300 and $90 \mathrm{~K}$ using either ArF pulsed laser $\left[\lambda_{\mathrm{exc}}=193 \mathrm{~nm}\right.$ (20 ns); Lumonics, EX-742] with a repetition rate of $10 \mathrm{~Hz}$ or a Nd:YAG (yttrium aluminum garnet) pulsed laser $\left[\lambda_{\mathrm{exc}}=266 \mathrm{~nm},(8 \mathrm{~ns})\right.$; Spectra Physics, GCR-190] with a repetition rate of $30 \mathrm{~Hz}$ as sources of excitation. The laser beams were focused by a $30-\mathrm{cm}$ lens into a $0.03-\mathrm{cm}^{2}$ spot. The samples were oriented to the laser beam by $45^{\circ}$. The PL was collected in a conventional $90^{\circ}$ geometry by a $0.5-\mathrm{m}$ SpectraPro-500 monochromator (Acton Research Corporation) with 1200 lines/mm grating blazed for the $500 \mathrm{~nm}$. The spectra were recorded in the time-resolved detection mode using an R943-02 photomultiplier (peak wavelength 300-800 nm; Hamamatsu) and gated electronics (SRS-250, Stanford Research System). The conventional scan spectra were measured using a CCD camera (Princeton Instruments, $330 \times 1100$ pixels) within an accumulation time of $2 \mathrm{~s}$. A set of optical filters has been used in order to cut stray radiation from lasers and scattered laser light from the samples. A LeCroy oscilloscope was used to measure the PL decay time constants.

\section{EXPERIMENTAL RESULTS}

\section{A. PL spectra for silica nanoparticles}

Prior to discussing the time-resolved dynamics of PL from silica nanoparticles, let us consider the spectra measured in

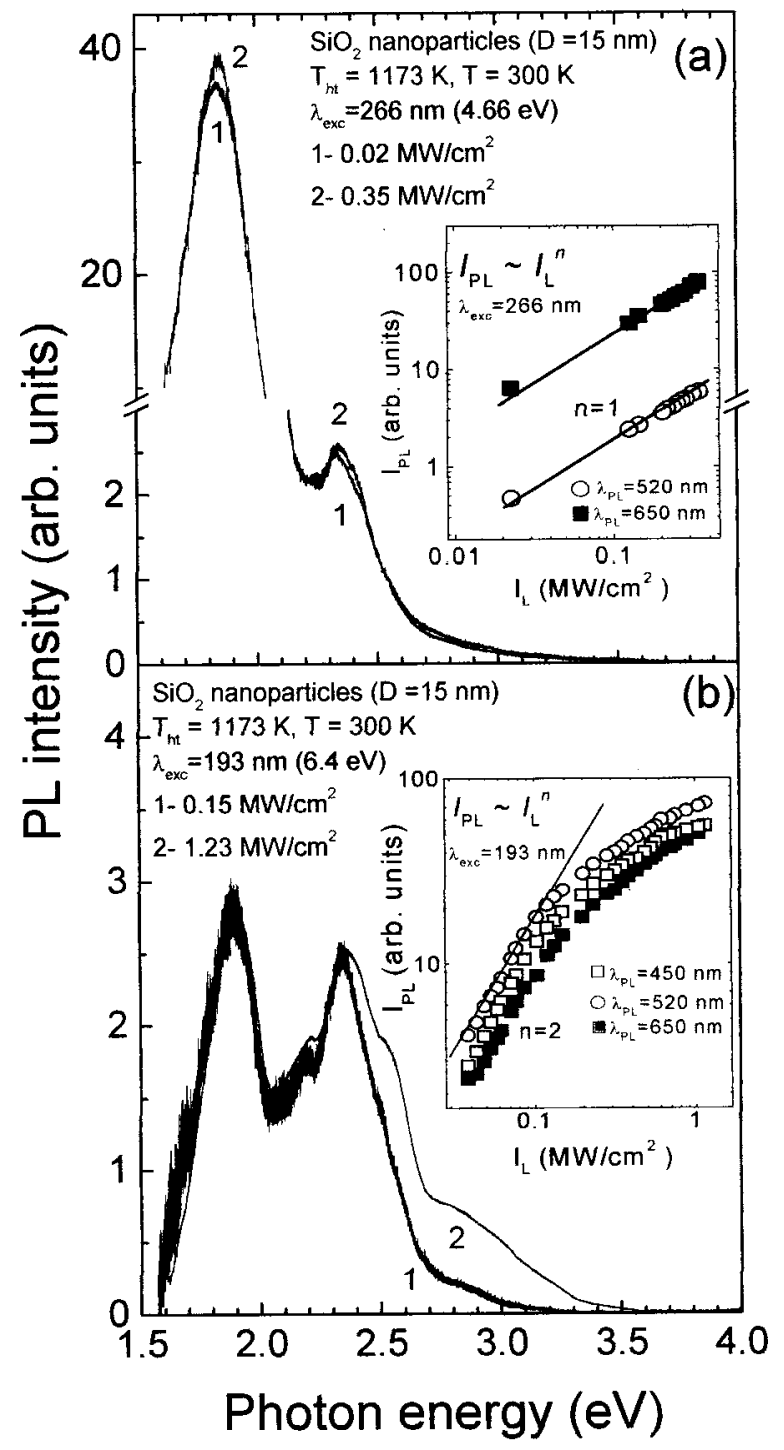

FIG. 1. Arbitrary normalized conventional scan measured PL spectra for $15-\mathrm{nm}$ silica particles $\left(T_{\mathrm{ht}}=1173 \mathrm{~K}\right)$ taken with different intensities of 4.66-eV (a) and 6.4-eV (b) laser light: (a) 1-0.02 and $2-0.35 \mathrm{MW} / \mathrm{cm}^{2}$; (b) $1-0.15$ and $2-1.23 \mathrm{MW} / \mathrm{cm}^{2}$. The insets show the corresponding intensity dependence of the PL yields for different PL bands. Solid lines are drawn as a guide to the eye showing the index $n$ of the power function $I_{\mathrm{PL}} \sim I_{L}^{n}$ on a log-log scale.

conventional detection mode using a CCD camera. We have used the power excitation with either 266-nm (4.66-eV) or 193-nm (6.4-eV) laser light. Figure 1 shows that the PL spectra measured with these two excitation regimes are considerably different. The red PL band peaked at $\sim 1.9 \mathrm{eV}$ is dominant for spectra measured with 4.66-eV excitation [Fig. 1 (a) ]. At the same time, the green PL band peaked at $\sim 2.35$ $\mathrm{eV}$ appears with a relatively small amplitude. When 6.4-eV light is applied, the red and green PL bands become comparable in intensity. Additionally, a broad PL band clearly manifests itself in the blue spectral range, which is strongly overlapped with 2.35-eV band [Fig. 1(b)]. Moreover, the 
relative intensity of blue PL increases with powering 6.4-eV laser light, whereas the spectra taken with $4.66-\mathrm{eV}$ excitation are practically the same within the broad range of laser light intensities used. The insets in Figs. 1(a) and 1(b) show the corresponding intensity dependences of the PL yields for the red, green, and blue bands. Since the PL intensity $\left(I_{\mathrm{PL}}\right)$ is known to be proportional to the laser light intensity $\left(I_{L}\right)$ in power $n$, where $n$ denotes the number of photons taken part in the excitation process, the PL yields versus the laser light intensities are plotted on a log-log scale. The slope of the intensity dependences for the red and green PL induced with 4.66-eV photons is $n=1$, thus indicating the one-photon excitation. Alternatively, if $6.4-\mathrm{eV}$ photons are applied, the two-photon excitation process occurs for all the PL bands, which tends to be saturated at higher laser light intensities.

We have previously suggested that the red and green PL from silica nanoparticles induced with $4.66-\mathrm{eV}$ photons is due to the direct excitation of NBOHC's and hydrogenrelated species, respectively. ${ }^{1-3,15}$ By contrast, the red and green light emitters are excited indirectly through the radiationless relaxation of TP-produced FE's in the case of 6.4-eV laser light. ${ }^{2,3,15}$ Additionally, the laser heating of FE's, which is due to their elastic scattering by the nanoparticle boundary in the presence of an intense laser field, results in the creation of the electron-hole plasma inside of each of the nanoparticles. Owing to plasma cooling once the laser pulse was terminated, the carriers may be condensed into the STE state. $^{2,3}$ The dynamics discussed can be imagined as an overcome of the laser-heated FE's through the STE barrier with subsequent radiative relaxation resulting in the blue emission assigned to STEPL. Therefore, we explain the aforementioned increase in the blue PL intensity with powering 6.4-eV light [Fig. 1(b)] as being due to an increase in the rate for FE's to overcome through the STE barrier. This experimental fact provides a proof that the origin of the blue emission band is different to those of red and green bands and is not related to impurities and structure defects. It is apparent that the blue band is absent in spectra taken with $4.66-\mathrm{eV}$ excitation because the total energy of two laser photons ( 9.32 $\mathrm{eV}$ ) is not enough to produce FE's [10.2 eV (Ref. 6)]. The direct TP excitation of STE's in silica is unlikely because of a strong lattice distortion for the STE state, resulting in a large shift of the potential energy surface with respect to that corresponding to the undisturbed lattice site. ${ }^{2}$

The time-resolved PL spectra measured for silica nanoparticles with 6.4-eV excitation at room temperature reveal the aforementioned red, green, and blue PL bands, which now can be deconvoluted by measuring spectra at different delay times (Fig. 2). The 2.35-eV band measured in conventional detection mode manifests a vibronic progression with an origin at $\sim 2.55 \mathrm{eV}$ and a spacing of about $0.165 \mathrm{eV}(1330$ $\mathrm{cm}^{-1}$ ) [Figs. 1(b) and 2]. The progression becomes poorly detectable in time-resolved spectra. We associate this fact with a significant loss in the signal intensity due to detection with long delay times. This band was assigned to hydrogenrelated species in our previous studies. ${ }^{1-3}$ The exact structure of green light emitters remains unknown, but many experimental facts indicate that hydrogen is involved in the emission process. ${ }^{1,2}$ Nevertheless, other species types like sil-

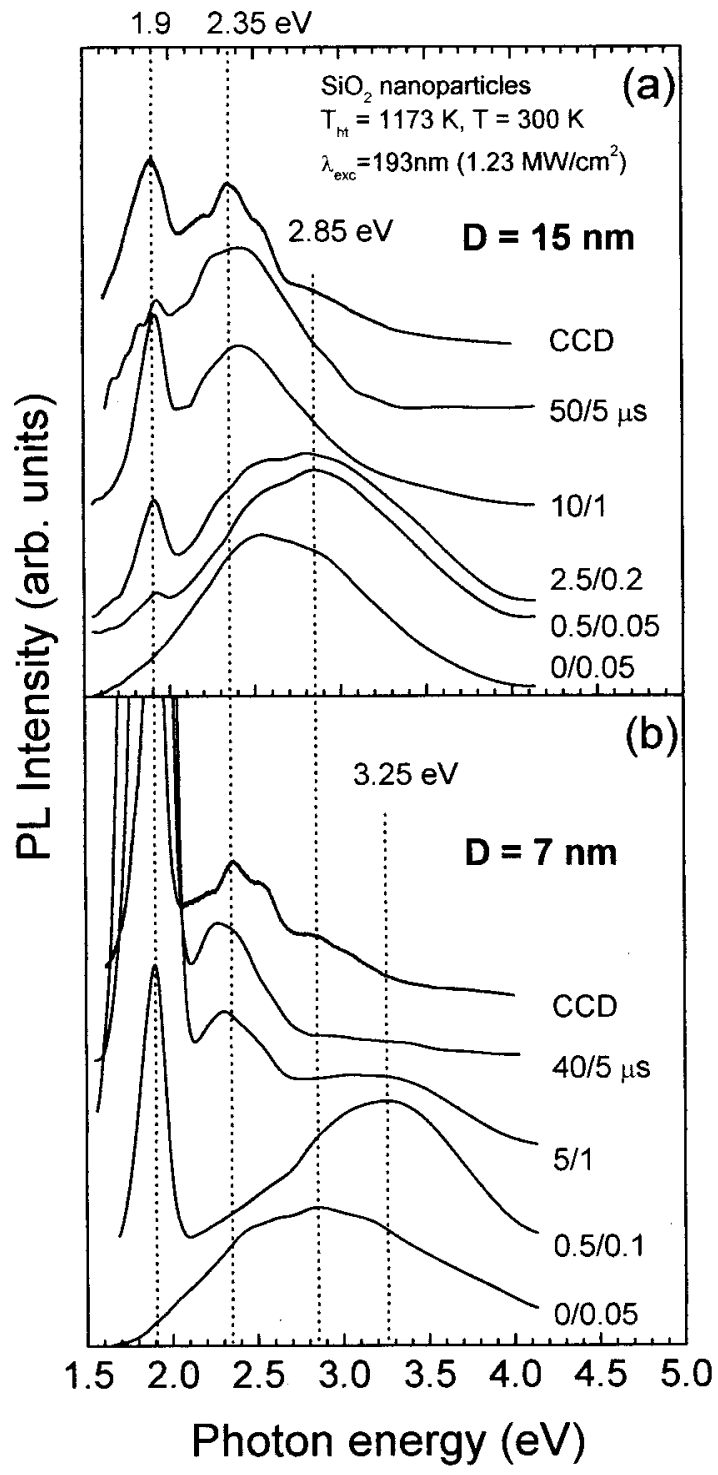

FIG. 2. Arbitrary normalized time-resolved PL spectra at room temperature for $15-\mathrm{nm}$ (a) and 7-nm (b) silica particles $\left(T_{\mathrm{ht}}\right.$ $=1173 \mathrm{~K})$ as compared to those measured in the conventional scan mode using CCD camera: $\lambda_{\text {exc }}=193 \mathrm{~nm}, I_{L}=1.23 \mathrm{MW} / \mathrm{cm}^{2}$. Numbers indicate the gate delay and gate width in microseconds, respectively. The individual spectrum baselines are arbitrarily shifted for better observation.

anone and dioxasilyrane were recently considered theoretically as potential green light emitters in silicon oxides. ${ }^{16}$ As we will show in Sec. III B, a similar PL band appears in hydrogen-containing bulk silica as well. This fact provides an additional support to the hydrogen-related nature of green light emitters. It has been suggested that the vibronic coupling in hydrogen-related species resulting from the interaction of electronic transitions with $\equiv \mathrm{Si}-\mathrm{H}-\mathrm{O}-\mathrm{Si}$ $\equiv$ bending vibrations in the ground electronic state is responsible for the progression observed. ${ }^{1,2}$

Figure 2 also shows the time-resolved PL spectra as compared to those measured by a CCD camera in the conventional detection mode. It can easily be seen that the PL spec- 
tra detected in conventional scans closely match those taken in time-resolved measurements, beginning with delay times longer than $10 \mu \mathrm{s}$. This peculiarity is due to the fact that the CCD camera is an integrated device, so the long-lived components contribute to the measured spectrum with higher efficiency during the given acquisition time. The decay-time constant for the 2.35-eV PL band is measured as $\sim 12 \mu \mathrm{s}$ $(300 \mathrm{~K})$ and $\sim 30 \mu \mathrm{s}(90 \mathrm{~K})$. As a result, the more short-lived blue PL measured in the conventional detection mode manifests itself with small amplitudes [Figs. 1(b) and 2]. Note that practically identical PL spectra have been measured with cathodoexcitation for thin thermally grown $\mathrm{SiO}_{2}$ films on a Si substrate, where the CCD camera was also used. ${ }^{13}$ The distinction between the CCD-measured spectra (Figs. 1 and 2) and those presented in Ref. 13 is the existence of a vibronic progression for the $2.35-\mathrm{eV}$ PL band in our measurements. Despite the similarity in a full width at half maximum (FWHM) for the green PL bands taken in both measurements, the different assignments have been suggested: we associate this PL band with hydrogen-related species, but the authors of Ref. 13 pointed to the STE nature of this band. Also note that the temperature lowering to $90 \mathrm{~K}$ increases the quantum efficiency of light emitters; however, it does not significantly affect the aforementioned time-resolved dynamics of PL spectra (Figs. 2 and 3).

The green PL band measured with 4.66-eV laser light decays much faster and practically disappears with delay time longer than 3-4 $\mu$ s (Figs. 4 and 5). On the strength of this fact we conclude that the nature of green light emitters excited with 6.4- and 4.66-eV light is different. One can suggest that both kinds of green light emitters are associated with hydrogen impurities, but they are involved in the complexes of different local geometries. This conclusion is consistent with the fact that the vibronic progression for the green band is characterized by different spacings when 6.4or 4.66-eV laser light is applied. ${ }^{1,2,15}$

The red PL band induced with 6.4-eV photons is normally peaked at $\sim 1.9 \mathrm{eV}$, and allows for a small variation between 1.89 and $1.93 \mathrm{eV}$ for different specimens (Figs. 2 and 3), which is due to the band inhomogeneous broadening. ${ }^{17}$ The lifetime for $1.9-\mathrm{eV}$ light emitters in combination with the band position and its FWHM is typical for NBOHC's in bulk silica. ${ }^{1,2,15,16}$ However, conventional scan measurements with 4.66-eV excitation have revealed the existence of the band shift toward the longer wavelength range. ${ }^{1,2}$ We previously suggested that this shift is due to the contribution from the second red band peaked at $\sim 1.79 \mathrm{eV}$, which was assigned to surface NBOHC's. ${ }^{1,2,15}$ The formation of NBOHC's has been shown to originate from the heat-pretreatment-induced dehydroxylation process of bulk and surface $\equiv \mathrm{Si}-\mathrm{OH}$ species. ${ }^{1,2}$ In particular, the assignment of the $1.79-\mathrm{eV}$ band is based on the fact that the rise of the band intensity with $T_{\mathrm{ht}}$ is inversely proportional to the surface hydroxyl concentration decrease. ${ }^{18}$ These assignments were recently tested by $a b$ initio calculation, and a good agreement with experimental data was obtained. ${ }^{19,20}$ The energetic difference between bulk and surface NBOHC's was suggested to result from an incorporation of non-bridging oxygen atoms into undistorted

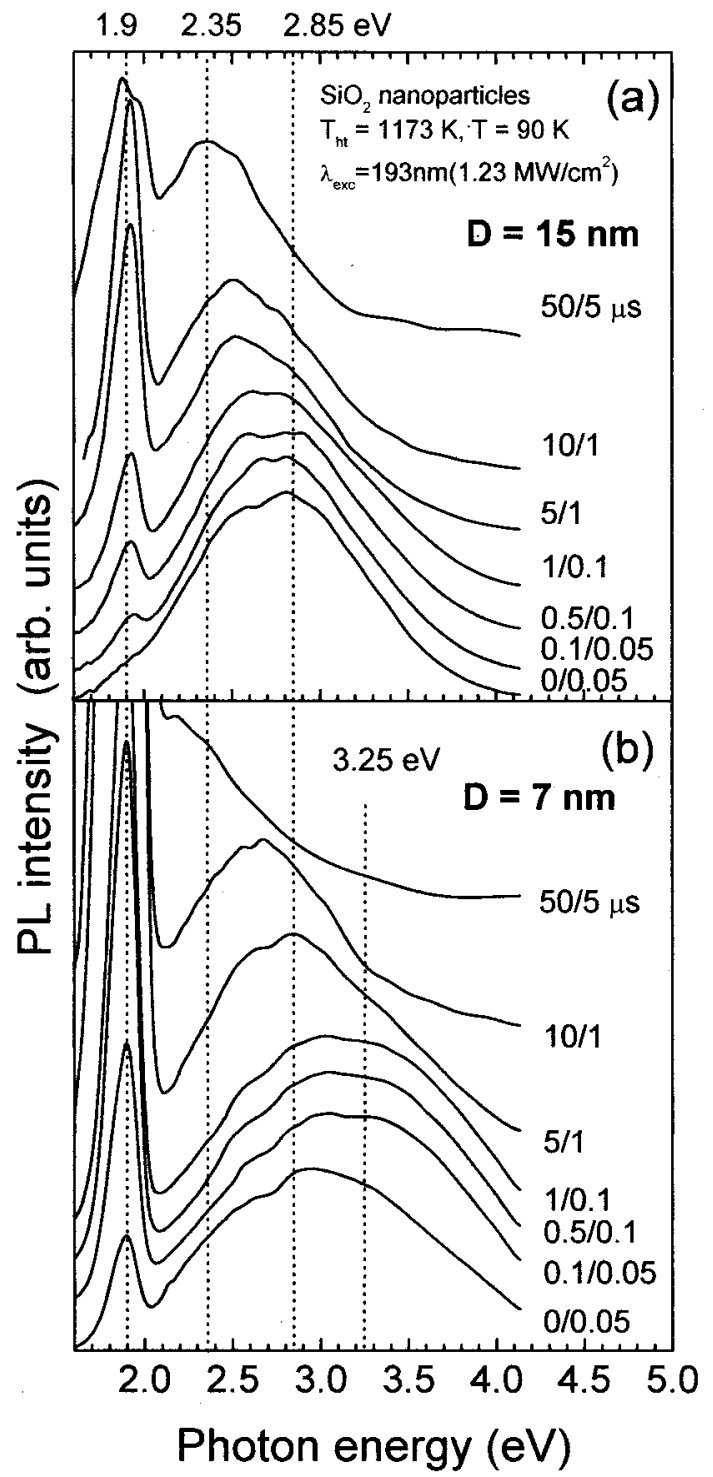

FIG. 3. Arbitrary normalized time-resolved PL spectra at $90 \mathrm{~K}$ for $15-\mathrm{nm}$ (a) and $7-\mathrm{nm}$ (b) silica particles $\left(T_{\mathrm{ht}}=1173 \mathrm{~K}\right): \lambda_{\text {exc }}$ $=193 \mathrm{~nm}, I_{L}=1.23 \mathrm{MW} / \mathrm{cm}^{2}$. Numbers indicate the gate delay and gate width in microseconds, respectively. The individual spectrum baselines are arbitrarily shifted for better observation.

$\mathrm{SiO}_{4}$ tetrahedrons (bulk species) and into distorted tetrahedrons creating twofold $\mathrm{Si}-\mathrm{O}$ rings (surface species). ${ }^{19,20}$

The time-resolved spectra shown in Figs. 4 and 5 substantiate our conclusion about the two red PL bands. Since the decay time constant for light emitters responsible for the 1.9-eV PL band $[\sim 8 \mu \mathrm{s}(300 \mathrm{~K})$ and $\sim 15 \mu \mathrm{s}(90 \mathrm{~K})]$ is smaller than that for the $1.79-\mathrm{eV}$ PL band $[\sim 40 \mu \mathrm{s}(300 \mathrm{~K})$ and $\sim 1 \mathrm{~ms}(90 \mathrm{~K})]$, there exists a shift of the compound red PL band as a whole toward the lower energy range with increasing delay time. The effect manifests itself most noticeably in the low-temperature PL spectra measured for 15-nm nanoparticles (Figs. 4 and 5). We explain the latter feature as being due to the higher concentration of surface NBOHC's in the composites of $15-\mathrm{nm}$ particles. This conclusion is also consistent with the fact that the concentration of 


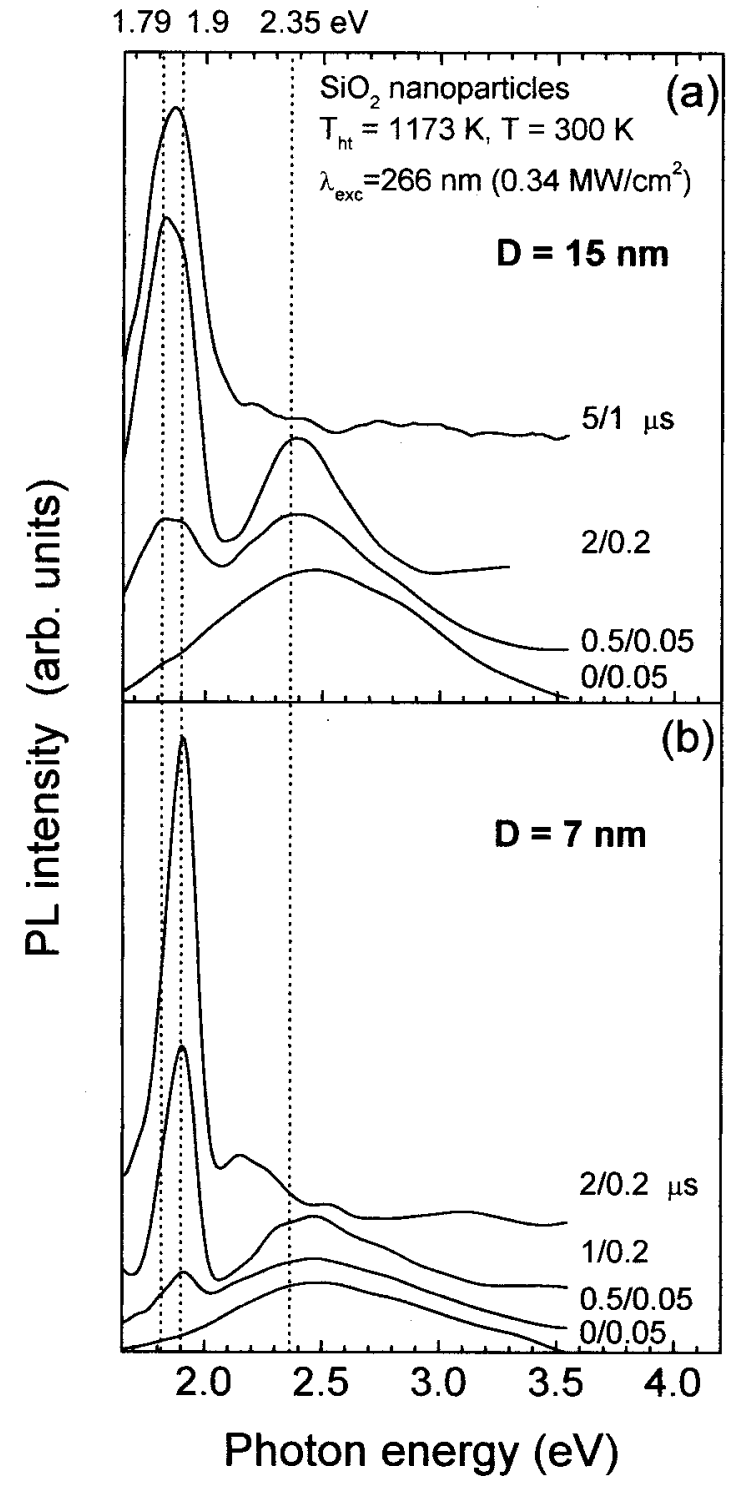

FIG. 4. Arbitrary normalized time-resolved PL spectra at room temperature for 15-nm (a) and 7-nm (b) silica particles $\left(T_{\mathrm{ht}}\right.$ $=1173 \mathrm{~K}): \lambda_{\mathrm{exc}}=266 \mathrm{~nm}, I_{L}=0.34 \mathrm{MW} / \mathrm{cm}^{2}$. Numbers indicate the gate delay and gate width in microseconds, respectively. The individual spectrum baselines are arbitrarily shifted for better observation.

surface hydroxyls for 7-nm particles is much less than that for $15-\mathrm{nm}$ particles. ${ }^{2}$ Note that the $1.9-\mathrm{eV}$ PL band measured with 4.66-eV laser light shows a similar decay-time constant as compared to that observed with 6.4-eV excitation, thus indicating that the bulk NBOHC's are responsible for light emission with both excitation regimes used.

The time-resolved PL spectra taken with 6.4-eV excitation (Figs. 2 and 3) and 4.66-eV excitation (Figs. 4 and 5) support the conclusion previously made about the mechanisms of the red PL excitation. Since the 4.66-eV photon energy coincides very closely with the absorption peak of NBOHC's $[\sim 4.8 \mathrm{eV}$ (Ref. 17)], both kinds of species located inside nanoparticles and on their surfaces are able to emit light in this case. $^{1,2,15,19,20}$ Correspondingly, an ordinary direct onephoton process occurs with this excitation regime. Alterna-

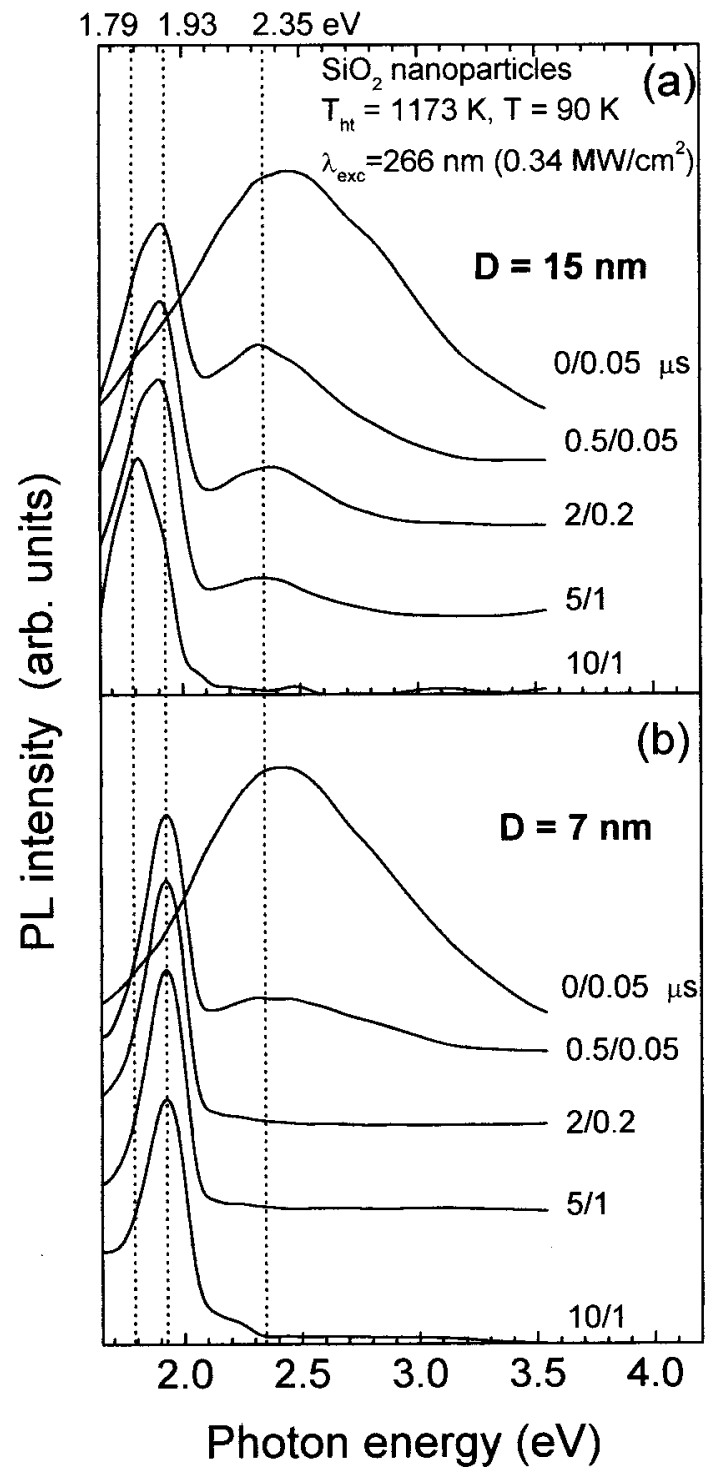

FIG. 5. Arbitrary normalized time-resolved PL spectra at $90 \mathrm{~K}$ for $15-\mathrm{nm}$ (a) and 7-nm (b) silica particles $\left(T_{\mathrm{ht}}=1173 \mathrm{~K}\right): \lambda_{\mathrm{exc}}$ $=266 \mathrm{~nm}, I_{L}=0.34 \mathrm{MW} / \mathrm{cm}^{2}$. Numbers indicate the gate delay and gate width in microseconds, respectively. The individual spectrum baselines are arbitrarily shifted for better observation.

tively, the 6.4-eV light excites only bulk NBOHC's because of an indirect mechanism of excitation involving the generation of TP-produced FE's followed by a FE energy transfer to NBOHC's. ${ }^{2,3,15}$ Since the interaction of FE's with surface NBOHC's is expected to be without energy transfer, ${ }^{2}$ one can only observe a single red PL band $(1.9 \mathrm{eV})$ assigned to bulk NBOHC's when 6.4-eV light is applied.

The blue band in the PL spectra measured in the conventional detection mode manifests itself as a shoulder near the 2.35-eV PL band (Figs. 1 and 2), and cannot be exactly identified without time-resolved techniques. The time-resolved spectra allow one to distinguish the blue PL band as being peaked at $\sim 2.85$ and $\sim 3.25 \mathrm{eV}$ for 15 - and 7-nm particles, respectively (Figs. 2 and 3). The decay-time constant for blue emission is found to be $\sim 5 \mu \mathrm{s}(300 \mathrm{~K})$ and $\sim 10 \mu \mathrm{s}(90$ 


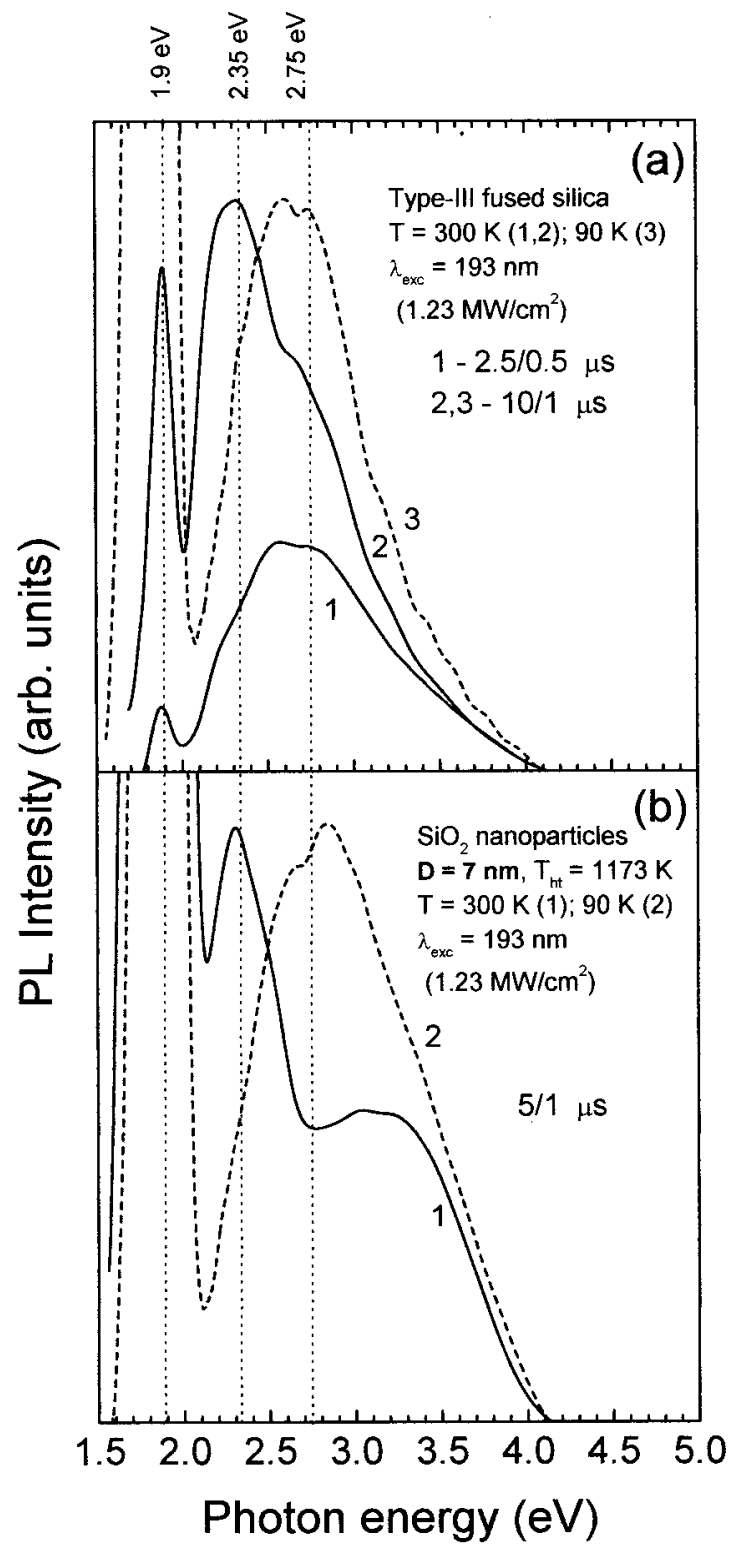

FIG. 6. Arbitrary normalized time-resolved PL spectra at roomtemperature $(1,2)$ and $90 \mathrm{~K}$ (3) for type-III fused silica (a) and at room temperature (1) and $90 \mathrm{~K}(2)$ for $7-\mathrm{nm}$ silica particles $\left(T_{\mathrm{ht}}\right.$ $=1173 \mathrm{~K}$ ) (b) $\lambda_{\text {exc }}=193 \mathrm{~nm}, I_{L}=1.23 \mathrm{MW} / \mathrm{cm}^{2}$. Numbers indicate the gate delay and gate width in microseconds, respectively.

$\mathrm{K}$ ), that is, less than those for the green and red PL bands. For the reason mentioned above about the CCD-measured spectra, the relative intensity of the blue PL band measured in the conventional detection mode is small, limiting the peak and profile of the PL band to be exactly determined. Since the 6.4-eV laser light can exclusively induce the blue PL when the TP regime of the PL excitation is achieved (Fig. 1), the blue band is assigned to STEPL. By contrast, the time-resolved spectra measured with $4.66-\mathrm{eV}$ excitation clearly show the absent of any blue PL with the aforementioned decay-time constants (Figs. 4 and 5). The blueshift of the STEPL band with decreasing nanoparticle size is suggested to result from the size effect in STEPL. ${ }^{3}$ The activation of hot (phonon-assisted) electronic transitions in the

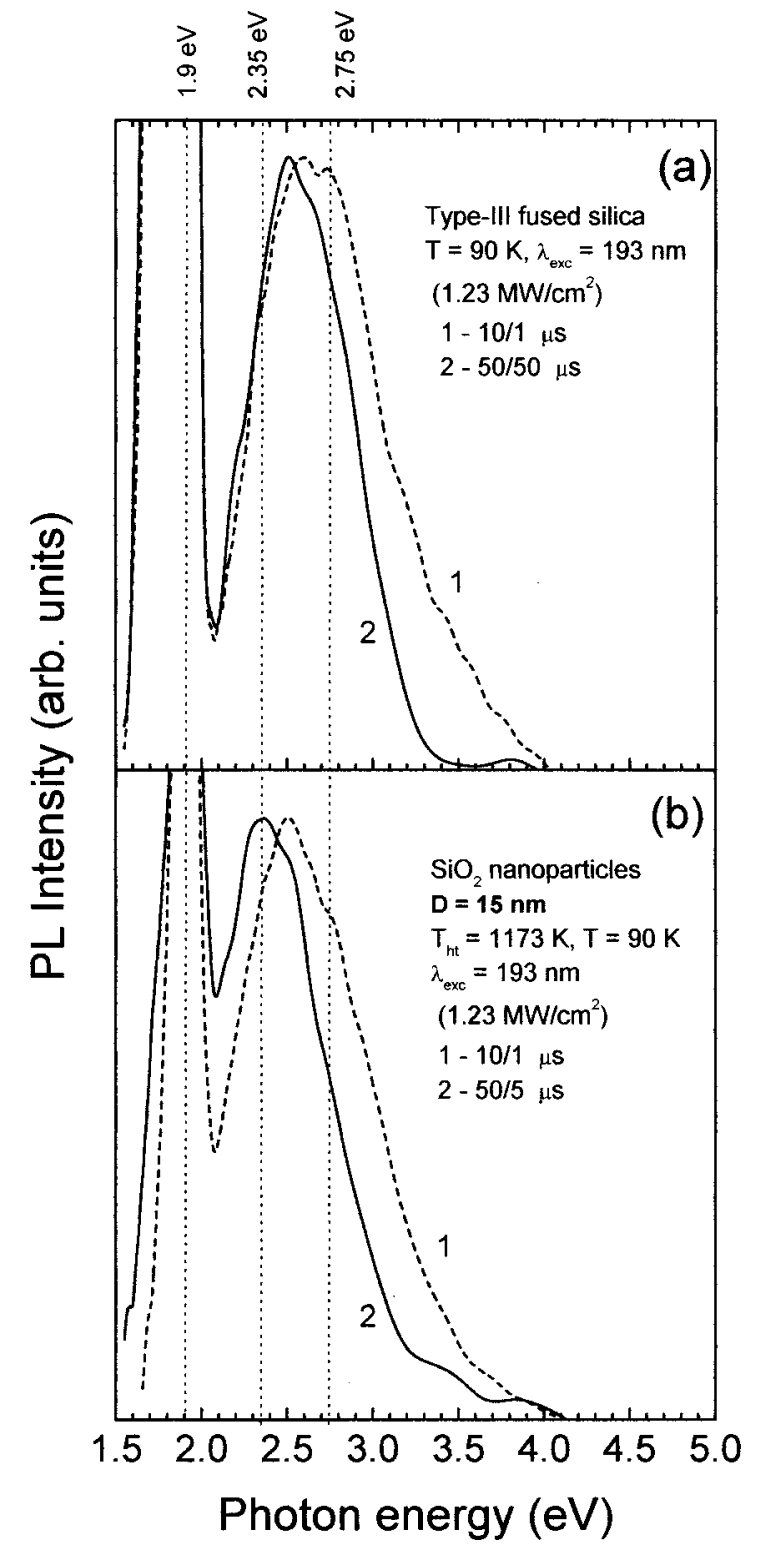

FIG. 7. Arbitrary normalized time-resolved PL spectra at $90 \mathrm{~K}$ for type-III fused silica (a) and $15-\mathrm{nm}$ silica particles $\left(T_{\mathrm{ht}}\right.$ $=1173 \mathrm{~K}$ ) (b) measured at different delay times: 1-10.0 and $2-50.0 \mu \mathrm{s} ; \lambda_{\mathrm{exc}}=193 \mathrm{~nm}, I_{L}=1.23 \mathrm{MW} / \mathrm{cm}^{2}$.

STE radiative relaxation involves more energetic phonons and their combinations with decreasing nanoparticle size. As a result, the STEPL band is extended and shifted toward the blue. ${ }^{3}$ This feature is also consistent with the idea that the blue emission is related to STE's.

Some additional specific feature of the time-resolved PL spectra measured for silica nanoparticles with 6.4-eV excitation appears as a nonmonotonic evolution of the blue PL intensity with delay time. The PL intensity rises up in a range of several microseconds beyond the excitation pulse and then decays with a constant of $\sim 5 \mu$ s (Fig. 2). Cooling to $90 \mathrm{~K}$ makes the effect much less detectable (Fig. 3), whereas the decay time becomes longer $(\sim 10 \mu \mathrm{s})$. A similar behavior of the STEPL band was recently reported for mesoporous silicas, which are characterized by extremely thin silica frag- 
ments of $\sim 1$-nm width (walls among pores). ${ }^{15}$ The effect was associated with the peculiarities of laser-heated FE's to overcome the STE barrier. ${ }^{3}$ The laser heating of FE's is suggested to be due to an scattering of FE's by the boundary of confined regions in mesoporous silica in the presence of a strong electromagnetic wave. ${ }^{3}$ Despite the fact that the peculiarities of the FE laser heating process are still to be clarified, the spectroscopic feature discussed again points out the excitonic nature of the blue PL band observed.

The conception of FE laser heating can also explain the shorter decay time of the STEPL from silica nanoparticles in comparison with that from bulk silica $(\sim 1 \mathrm{~ms})$. Because of an increase in the efficiency of the STE barrier overcome for laser-heated FE's, ${ }^{3}$ they relax into the STE states much faster. In other words, the high density of electron-hole plasma created in silica nanoparticles results in the higher efficiency of STE and STBE state formation as compared to the situation occurring in bulk silica, where the FE's excited are not heated and probably penetrate the STE barrier by tunneling. This conclusion takes into account the fact that the total decay-time constant for STEPL consists of two components resulting from the time required for FE's to be trapped into the STE state and an intrinsic lifetime of STE's. ${ }^{2,15}$

\section{B. PL spectra for type-III fused silica}

In this section we present the experimental results of a PL study of bulk type-III fused silica as compared to those discussed in Sec. III A for silica nanoparticles. Figures 6(a) and 7(a) show the time-resolved PL spectra for type-III fused silica, which were measured with $6.4-\mathrm{eV}$ excitation at 300 and $90 \mathrm{~K}$. It should be noted that both 4.66- and 6.4-eV light irradiations of the sample lead to the formation of bulk NBOHC's. However, the efficiency of the NBOHC formation is much higher with 6.4-eV laser light. This fact is not reconcilable with silica nanoparticles, where the radiationinduced NBOHC's have exclusively been observed for smaller-sized particles $(7 \mathrm{~nm})$ with 6.4-eV excitation. ${ }^{2}$ The corresponding 1.9-eV PL band for bulk silica used grows in intensity within the initial stage of irradiation and then saturates. The latter condition was chosen for measuring the time-resolved PL spectra. All parameters for the red PL band exactly correspond to those discussed for silica nanoparticles in the preceding section and have commonly been measured for bulk $\mathrm{SiO}_{2}$ materials. ${ }^{17}$ The process of NBOHC formation is more efficient at low temperature (Figs. 6 and 7), and is known to result from the STBE radiationless relaxation (see Refs. 2 and 9, and references therein). Since the type-III fused silica is a hydrogen-containing material, we also consider the processes of $\mathrm{NBOHC}$ formation due to the radiolytic reaction ${ }^{21}$

$$
\equiv \mathrm{Si}-\mathrm{OH} \stackrel{6.4 \text { or } 4.66 \mathrm{eV}}{\longrightarrow} \equiv \mathrm{Si}-\mathrm{O} \cdot+\cdot \mathrm{H} .
$$

In order to discriminate these two processes, one can probe the STEPL band. We will associate the excitonic mechanism of NBOHC formation with the process appearing simultaneously with long-lived STEPL. By contrast, if the STEPL band is absent, the NBOHC formation process is assumed to be due to the radiolytic reaction. Also, we do not discuss all short-lived responses in PL from type-III fused silica (with a decay time of less than $1 \mu \mathrm{s}$ ), assuming that they result from one-photon-excited uncontrolled impurities.

Totally, the PL spectra measured with 6.4-eV excitation are similar to those typically observed for crystalline and glassy $\mathrm{SiO}_{2}$ with band-to-band excitation. ${ }^{4-8,10-13}$ It can easily be seen that the PL spectra taken with the delay time longer than $1 \mu \mathrm{s}$ also resemble those that have been measured for silica nanoparticles (Figs. 6 and 7). In the same way, there is an overlap between the green and blue PL bands. We propose that the origin of these bands is similar to that established for silica nanoparticles, that is, to hydrogenrelated species and STE's, respectively. On the other hand, the STEPL band is known to commonly peak for bulk crystalline $\mathrm{SiO}_{2}$ materials at $2.75 \mathrm{eV}$ with a FWHM of $\sim 0.7$ $\mathrm{eV}^{4-8}$ However, this band is much broader, and is peaked at $\sim 2.35 \mathrm{eV}$ in amorphous silicon dioxide. ${ }^{10-13}$ The corresponding FWHM of the compound blue-green PL band for type-III fused silica measured in the current work is in good agreement with previous data for crystalline $\mathrm{SiO}_{2} \cdot{ }^{4-8} \mathrm{Nev}$ ertheless, the lifetime of green light emitters $(\tau \sim 1 \mathrm{~ms}$ at 90 $\mathrm{K})$ corresponds to that typically observed for STEPL in amorphous materials. Note that the measurements of timeresolved spectra with delay time longer that $50 \mu$ s were limited by extremely low intensity of the signal, despite the fact that the exponential PL decay can still be monitored by an oscilloscope.

Similarly as for nanoparticles, the 1.9- and $2.35-\mathrm{eV}$ PL bands become relatively more intense with increasing delay time in room temperature measurements [Fig. 6(a), curves 1, and 2], indicating that the lifetime of the red and green light emitters is longer than that related to STE's. However, the low-temperature spectrum taken with the same delay time reveals a shift of the 2.35-eV PL band toward the higherenergy range [Fig. 6(a), curves 2 and 3]. We explain this shift as being due to a decrease in the green PL intensity. Since the vibronic progression for $2.35-\mathrm{eV}$ PL band started at $\sim 2.55$ $\mathrm{eV}$, only the peak corresponding to the progression origin manifests itself in the spectrum measured at $90 \mathrm{~K}$. The decrease of the green PL intensity with temperature lowering is due to a decrease in the rate of the FE energy transfer to hydrogen-related species. This is also consistent with the fact that the self-trapping rate for FE's increases with temperature lowering. ${ }^{6}$ As a result, the STEPL band becomes stronger at low temperature measurements [Fig. 6(a)]. A similar feature is observed for silica nanoparticles. Figure 6(b) shows examples of the corresponding time-resolved spectra taken for 7-nm silica nanoparticles. The lack of a FE energy transfer to light emitters, responsible for a $2.35-\mathrm{eV}$ PL band and an increase in the FE self-trapping rate, also explains the intensity redistribution between the green and blue PL band with temperature lowering.

Figure 7(a) shows that the relative intensity of the $2.75-\mathrm{eV}$ band measured for type-III fused silica at $90 \mathrm{~K}$ drops with increasing delay time. However, the intensity decrease is not as large as observed for room-temperature spectra [Fig. 6(a)], indicating that the lifetime of the blue light emitters increases when the temperature is lowered. Since the decay time for the $2.35-\mathrm{eV}$ PL band is longer than that 
for the 2.75-eV one, the green PL band tends to be stronger with increasing delay time. This appears as a narrowing of the compound blue-green PL band [Figs. 6(a) and 7(a)]. Additionally, there exists a shift of the compound band toward the lower-energy range, which mainly appears in roomtemperature measurements. As this takes place, the real peak position of the STEPL band does not change significantly as compared to that typically observed for crystalline $\mathrm{SiO}_{2}$. Note that the compound blue-green PL band was initially considered as a single band and assigned to STEPL. ${ }^{10} \mathrm{~A}$ similar narrowing and shift with increasing delay time occur for the compound blue-green PL band measured for silica nanoparticles [Figs. 2, 3, and 7(b)]. The shift is practically the same as that observed in room-temperature measurements for type-III fused silica [Figs. 6(a) and 7(b)]. Thus the peak of the green PL band can occur at two positions, either at $\sim 2.55$ or $\sim 2.35 \mathrm{eV}$, which correspond to different vibronic components of the progression shown in Fig. 1(b). Therefore, if we assume that the vibronic coupling in hydrogen-related species can vary with temperature and the local geometry of complexes, the shift of the green PL band can be explained as intensity redistribution among different components of the vibronic progression.

The PL spectra measured with $4.66-\mathrm{eV}$ laser light are shown in Fig. 8. Only short-lived emission components appear in the blue-green range, and are assumed to result from a direct one-photon excitation of uncontrolled impurities. As a result, the PL in this range disappears with delay time longer than 1-2 $\mu \mathrm{s}$, meanwhile, the NBOHC's contribute to emission with a typical decay-time constant. Therefore, we propose that the mentioned radiolytic reaction is a reason for NBOHC formation in type-III fused silica with 4.66-eV excitation. This reaction does not involve any process of exciton generation. In sharp contrast, the NBOHC formation with $6.4-\mathrm{eV}$ photon irradiation is dominated by the excitonic mechanism.

Note that only a single red PL band, peaked at $1.9 \mathrm{eV}$ and originating from bulk NBOHC's manifests itself with 4.66-eV excitation, pointing to a small concentration of surface NBOHC's as compared to that for silica nanoparticles. Thus the spectroscopic properties of silica nanoparticles and type-III fused silica are found to be close in general. However, the 1.79-eV PL band originating from surface NBOHC's additionally contributes to the PL from silica nanoparticles subjected to $4.66-\mathrm{eV}$ photon irradiation. The main difference between the PL spectra measured for silica nanoparticles and type-III fused silica with 6.4-eV excitation is a shift of the STEPL band toward the higher-energy range for nanoscale materials, resulting from the activation of phonon-assisted transitions within STE radiative relaxation.

\section{DISCUSSION}

On the strength of our experimental data taken with TP band-to-band excitation presented in preceding sections, let us discuss the nature of light emitters in amorphous $\mathrm{SiO}_{2}$ materials. First note that the mentioned overlap of the PL bands induced by the band-to-band excitation with ionizing radiation or energetic electrons has been observed for differ-

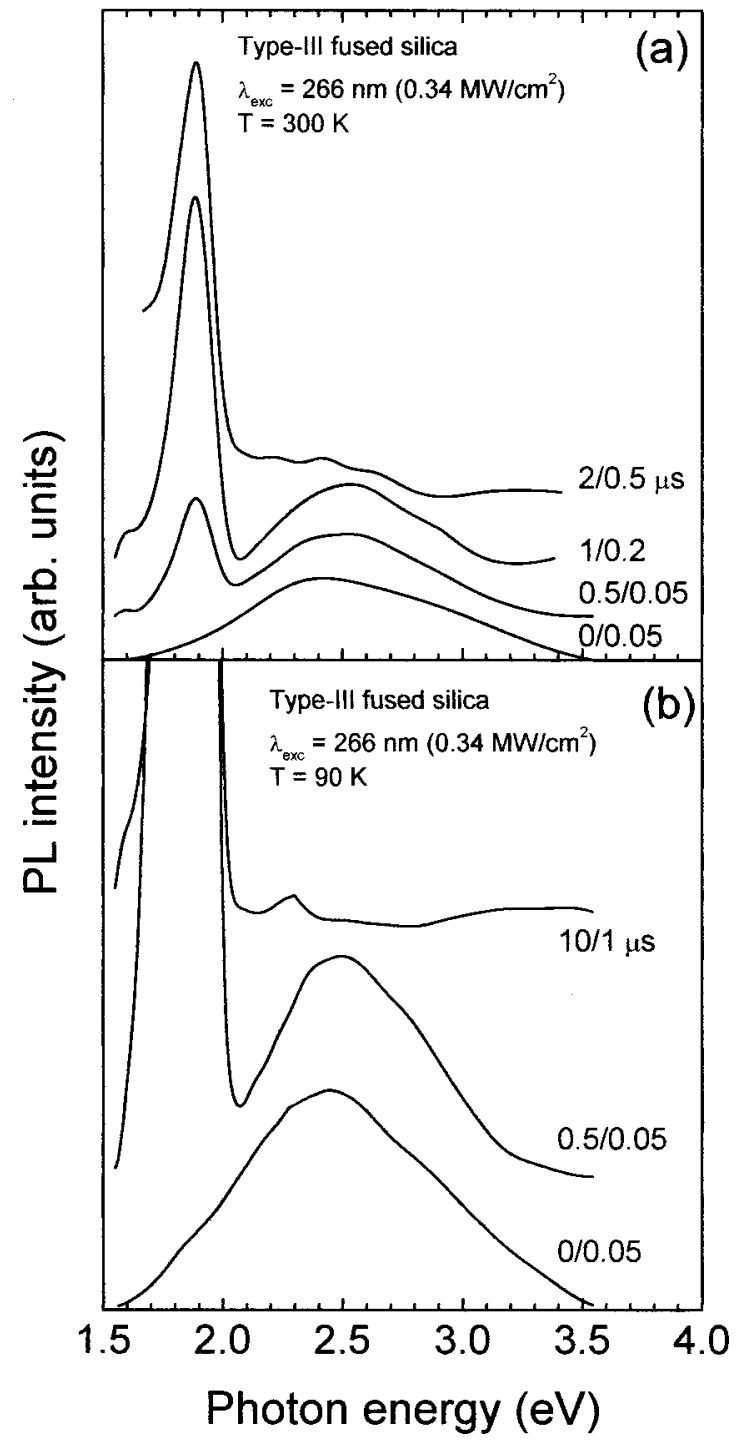

FIG. 8. Arbitrary normalized time-resolved PL spectra at room temperature (a) and $90 \mathrm{~K}$ (b) for the type-III fused silica: $\lambda_{\text {exc }}$ $=266 \mathrm{~nm}, I_{L}=0.34 \mathrm{MW} / \mathrm{cm}^{2}$. Numbers indicate the gate delay and gate width in microseconds, respectively. The individual spectrum baselines are arbitrarily shifted for better observation.

ent types of silicas. However, the PL bands were not spectroscopically deconvoluted, and have been considered as a single band peaked at $\sim 2.35 \mathrm{eV}$ and assigned to the STEPL. ${ }^{10-13}$ On the other hand, it is known that the STEPL band for crystalline $\mathrm{SiO}_{2}$ materials is peaked at $\sim 2.75 \mathrm{eV}^{4-8}$ As a result, many authors suggested that the STEPL band is placed at different positions for bulk crystalline and amorphous silicon dioxide and reflects the material specific features. However, the latter statement is not consistent with our experimental results presented in the current paper. We showed that the STEPL band in silica nanoparticles as well as in bulk silicas is located at practically the same spectral position as that for bulk crystalline $\mathrm{SiO}_{2}$. By contrast, the PL band peaked at $2.35 \mathrm{eV}$, which was initially assigned to STEPL from silicas, is associated with hydrogen-related species. The latter conclusion is in agreement with the model of hydrogen-stabilized STE in amorphous $\mathrm{SiO}_{2}$ materials. ${ }^{8}$ 
Our assignment of the blue PL band is based on the following experimental facts: (1) this PL band can exclusively be observed when the TP regime of PL excitation with 6.4-eV laser light is applied; (2) the relative intensity of the blue band as compared to the green and red PL bands (hydrogen-related centers and NBOHC's, respectively) increases with increasing laser light intensity, indicating an increase in the rate of the STE barrier overcome for laserheated FE's; (3) there exists a nonmonotonic behavior of the blue band with the delay time, which is consistent with the excitonic nature of the emission; (4) there exists a blue shift of the STEPL band with decreasing size of nanoparticles, which again points to excitons.

As an additional feature, which could lead to an incorrect assignment of $2.35-\mathrm{eV}$ PL band measured in conventional scans is the fact that the discussed blue and green PL bands are characterized by the nearby values of the decay-time constants. Since the decay time for the green PL is somewhat longer than that for the blue one, clearly that the peak of this compound band will be redshifted with increasing delay time. The hydrogen content in $\mathrm{SiO}_{2}$ materials will also affect the relative intensities of the blue and green PL bands. Thus any shift in the range between 2.3 and $2.8 \mathrm{eV}$ for the compound PL band, measured with band-to-band excitation and initially assigned to STEPL from bulk silicas, can be explained as an intensity redistribution between two bands related to STE's and hydrogen-related species.

Let us consider the experimental data from the initial paper, in which the 2.35-eV PL band observed for amorphous silicon dioxide with band-to-band excitation was assigned to STEPL. ${ }^{10}$ The authors of Ref. 10 measured the time-resolved PL spectra for amorphous $\mathrm{SiO}_{2}$, and observed a very broad PL band in the range of $1.5-3.3 \mathrm{eV}$ with a maximum at $\sim 2.35 \mathrm{eV}$. The peak of the band is slightly blueshifted as the delay time increases. The main experimentally based argument discussed by the authors in order to identify the nature of 2.35-eV PL band is the fact that identical decays for the transient absorption states related to STE's and the 2.35-eV emission were observed. However, for reasons not well understood, the authors have monitored the PL at $\sim 2.75 \mathrm{eV}$, the position of which is typical for the STE's in crystalline $\mathrm{SiO}_{2}$ materials, but the conclusion has been made for $2.35-\mathrm{eV}$ band as a whole. It is evident that from these results we can only conclude that the light emission at $2.75 \mathrm{eV}$ is related to STE's. If we assume the compound nature of the $2.35-\mathrm{eV}$ PL band observed by the authors, then the conclusion made in the paper mostly reflects the behavior of the $2.75-\mathrm{eV}$ band rather than the $2.35-\mathrm{eV}$ band. The progressive blueshift of the 2.35-eV PL band with increasing delay time discussed by the authors can also be explained as a result of intensity redistribution between two PL bands with different decay times as discussed for type-III fused silica in the current paper (Figs. 6 and 7).

Thus we suggest that the STEPL band for bulk amorphous and crystalline $\mathrm{SiO}_{2}$ manifests itself at similar spectral position around $2.75 \mathrm{eV}$. Let us consider how this conclusion is consistent with the model of the $\mathrm{STE}$ in $\mathrm{SiO}_{2}$ materials. It is known that the most stable forms of bulk $\mathrm{SiO}_{2}$ are constructed from vertex-sharing $\mathrm{SiO}_{4}$ tetrahedrons forming a continuous three-dimensional network. The $\mathrm{Si}-\mathrm{O}-\mathrm{Si}$ angles are flexible and vary between $125^{\circ}$ and $180^{\circ}\left(144^{\circ}\right.$ for the $\alpha$ quartz). The torsion angles are nonrigid as well, which results in the variety of $\mathrm{SiO}_{2}$ polymorphs. ${ }^{20,22}$ Meanwhile, the $\mathrm{SiO}_{4}$ tetrahedrons conserve their shape with practically unchanged Si-O distances and O-Si-O angles. ${ }^{20,22,23}$ The modification of the $\mathrm{SiO}_{2}$ network is expected to be due to rotations of vertex-sharing rigid tetrahedrons leading to essential shifts of oxygen atoms, while the positions of $\mathrm{Si}$ atoms remain nearly unchanged. The model of the STE in $\mathrm{SiO}_{2}$ takes into account an assumption that the electronic component of the STE is an $E^{\prime}$ center (oxygen vacancy, $\equiv \mathrm{Si}-\mathrm{Si} \equiv)$ and the hole component is a peroxy linkage $(\equiv \mathrm{Si}-\mathrm{O}-\mathrm{O}-\mathrm{Si} \equiv) .{ }^{4}$ Since there is a large Stokes shift of the STEPL band, a strong distortion of the $\mathrm{SiO}_{2}$ lattice accompanies the process of STE formation. It is known that the oxygen vacancy formation is most likely to be responsible for this lattice distortion as compared to the peroxy linkage. ${ }^{20}$ Because the $\mathrm{Si}-\mathrm{Si}$ distance is expected to be weakly dependent on the flexibility of both $\mathrm{SiO}_{4}$ tetrahedron torsion and $\mathrm{Si}-\mathrm{O}-\mathrm{Si}$ angles in three-dimensional $\mathrm{SiO}_{2}$ network, the structure of the STE should be insensitive to the different modifications of the $\mathrm{SiO}_{2}$ network. As a result, the peak position of the STEPL band for different $\mathrm{SiO}_{2}$ polymorphs is also expected to occur at similar spectral positions. However, a detailed knowledge of the formation of STE's and their light-emitting properties in different $\mathrm{SiO}_{2}$ polymorphs can be achieved by combining experimental data with high-level ab initio quantum-chemical modeling.

\section{CONCLUSIONS}

In summary, on the basis of time-resolved PL measurements with TP band-to-band excitation for silica nanoparticles in comparison with bulk type-III fused silica, we have provided evidence that the PL properties of silica nanoparticles closely correspond to those of bulk materials. However, in addition to the PL bands observed for bulk silica with 4.66-eV excitation, there exists a $1.79-\mathrm{eV}$ PL band originated from surface NBOHC's, indicating the large concentration of such species in nanoscale silica materials. The main difference between the PL spectra measured for silica nanoparticles and type-III fused silica with $6.4-\mathrm{eV}$ excitation is a shift of the STEPL band toward the higher-energy range for nanoscale materials, resulting from the unique behavior of FE's in the confined space of silica nanoparticles. The remaining 1.9- and 2.35-eV PL bands are peaked at similar positions for both nanoscale and bulk materials, and are assigned to bulk NBOHC's and hydrogen-related species, respectively.

We have concluded that the experimental data presented in Ref. 10 and initially used to identify the STEPL band in amorphous $\mathrm{SiO}_{2}$ materials can be explained as a result of contributions from two overlapping PL bands. Only one of these bands is related to STE's and situated at practically the same spectral position as that for bulk crystalline $\mathrm{SiO}_{2}$ $(\sim 2.75 \mathrm{eV})$. The redshift of the STEPL band observed for amorphous silicon dioxide discussed before in numerous publications, from our point of view, is due to the contribu- 
tion from another band peaked at $2.3-2.4 \mathrm{eV}$ and assigned to hydrogen-related centers. Because the larger concentration of hydrogen-related species is expected to be for amorphous materials, the PL band related to these centers becomes dominant.

\section{ACKNOWLEDGMENTS}

The authors acknowledge Academia Sinica, Taiwan National Science Council, and China Petroleum Corporation of Taiwan, Republic of China, for financial support.
*Corresponding author. Present address: Department of Physics and Astronomy, Vanderbilt University, Nashville, Tennessee 37235. Email address: yuri.d.glinka@ vanderbilt.edu

${ }^{1}$ Yu. D. Glinka, S. H. Lin, and Y.-T. Chen, Appl. Phys. Lett. 75, 778 (1999)

${ }^{2}$ Yu. D. Glinka, S. H. Lin, and Y.-T. Chen, Phys. Rev. B 62, 4733 (2000).

${ }^{3}$ Yu. D. Glinka, S. H. Lin, L. P. Hwang, Y.-T. Chen, and N. H. Tolk, Phys. Rev. B 64, 085421 (2001).

${ }^{4}$ D. L. Griscom, in Proceedings of the Thirty-Third Frequency Control Symposium (Electronics Industries Association, Washington, DC, 1979), p. 98.

${ }^{5}$ C. Itoh, K. Tanimura, N. Itoh, and M. Itoh, Phys. Rev. B 39, 11183 (1989).

${ }^{6}$ A. N. Trukhin, J. Non-Cryst. Solids 149, 32 (1992).

${ }^{7}$ W. Joosen, S. Guizard, P. Martin, G. Petite, P. Agostini, A. Dos Santos, G. Grillon, D. Hulin, A. Migus, and A. Antonetti, Appl. Phys. Lett. 61, 2260 (1992).

${ }^{8}$ M. A. Stevens Kalceff, Phys. Rev. B 57, 5674 (1998).

${ }^{9}$ L. Skuja, J. Non-Cryst. Solids 239, 16 (1998).

${ }^{10}$ K. Tanimura, C. Itoh, and N. Itoh, J. Phys. C 21, 1869 (1988).

${ }^{11}$ C. Itoh, T. Suzuki, and N. Itoh, Phys. Rev. B 41, 3794 (1990).

${ }^{12}$ M. Goldberg, H.-J. Fitting, A. Trukhin, J. Non-Cryst. Solids 220,
69 (1997).

${ }^{13}$ A. N. Trukhin, M. Goldberg, J. Jansons, H.-J. Fitting, and I. A. Tale, J. Non-Cryst. Solids 223, 114 (1998).

${ }^{14}$ N. Kuzuu, Y. Matsumoto, and M. Murahara, Phys. Rev. B 48, 6952 (1993).

${ }^{15}$ Yu. D. Glinka, S. H. Lin, L. P. Hwang, and Y.-T. Chen, J. Phys. Chem. B 104, 8652 (2000).

${ }^{16}$ A. S. Zyubin, A. M. Mebel, S. H. Lin, and Yu. D. Glinka, J. Chem. Phys. 116, 9889 (2002).

${ }^{17}$ L. Skuja, J. Non-Cryst. Solids 179, 51 (1994).

${ }^{18}$ Yu. D. Glinka, Zh. Eksp. Teor. Fiz. 111, 1748 (1997) [JETP 84, 957 (1997)].

${ }^{19}$ Yu. D. Glinka, A. S. Zyubin, A. M. Mebel, S. H. Lin, L. P. Hwang, and Y. T. Chen, Eur. Phys. J. D 16, 279 (2001).

${ }^{20}$ A. S. Zyubin, Yu. D. Glinka, A. M. Mebel, S. H. Lin, L. P. Hwang, and Y. T. Chen, J. Chem. Phys. 116, 281 (2002).

${ }^{21}$ H. Nishikawa, R. Nakamura, R. Tohmon, Y. Ohki, Y. Sakurai, K. Nagasawa, and Y. Hama, Phys. Rev. B 41, 7828 (1990).

${ }^{22}$ L. W. Hobbs, C. E. Jesurum, V. Pulim, and B. Berger, Philos. Mag. A 78, 679 (1998).

${ }^{23}$ Y. Le Page, L. D. Cavlert, and E. G. Gabe, J. Phys. Chem. Solids 41, 721 (1980). 